\title{
Investigation of the Clinical Efficacy and Dosage of Intravenous Ciprofloxacin in Patients with Respiratory Infection
}

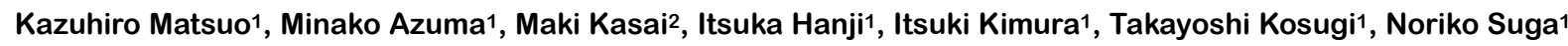 \\ and Mitsutoshi Satoh² \\ ${ }^{1}$ Department of Pharmacy, Toho University Omori Medical Center, 6-11-1 Omorinishi, Ota-ku, Tokyo 143-8540, \\ JAPAN; ${ }^{2}$ Department of Toxicology and Pharmacology, Faculty of Pharmaceutical Sciences, Toho University, 2-2-1 \\ Miyama, Funabashi, Chiba 274-8510, JAPAN
}

Received, October 3, 2008; Revised, December 12, 2008; Accepted, December 30, 2008, Published, December 30, 2008.

\begin{abstract}
Purpose. Multidrug-resistant Pseudo- monas aeruginosa (MDRP), which seems to be linked to antibiotics consumption, has become a significant problem. Infection control is of vital importance, hence, proper use of antimicrobial drugs is one of the most crucial roles of hospital pharmacists. In this study, we surveyed patients who had been prescribed intravenous (i.v.) ciprofloxacin (CPFX) as the only antibiotic, and evaluated the relation between the antimicrobial activity of CPFX and pharmacokinetic /pharmacodynamic from the blood concentration of CPFX. Methods. This study was performed retrospectively to 112 adult patients diagnosed as having respiratory infections who had been treated as inpatients with intravenous CPFX for more than 3 days at Toho University Omori Hospital in Tokyo. Minimum inhibitory concentration (MIC) for the bacterium was obtained from the antimicrobial susceptibility testing performed according to the guidelines. The potential efficacy of CPFX was evaluated by the peak plasma concentration (Cmax), area under the concentration curve (AUC) and AUC/MIC. The predictive AUC of each patient was obtained from the modified formulae reported by Forrest et al. (1993) [1]. Results. Although CPFX is excreted from the kidney, standard treatment with this drug does not take renal function into consideration. Our results indicated that CPFX was effective in less than $50 \%$ of the patients who received it. Moreover, the AUC/MIC ratio in both the responder group and the non-responder group was less than 125 that is the clinical target
\end{abstract}

ratio of CPFX for gram-negative bacteria. Conclusion. These results suggest that the clinical use of CPFX for the treatment of infectious diseases does not reach the target AUC/MIC ratio, and that the concentration of CPFX is not within the range to which many pathogens are susceptible in a large proportion of patients. To ensure the effective treatment of patients with infectious diseases and to prevent the development of resistance in bacteria, we recommend therapeutic drug monitoring (TDM) of CPFX in hospitals.

\section{INTRODUCTION}

Intravenous ciprofloxacin (CPFX) was the first antibacterial fluoroquinolone in Japan designed for injection. It was approved in September 2000 and marketed in November of the same year. CPFX has strong antibacterial activity against gram-positive and negative bacilli, and a wide antibacterial spectrum that includes atypical bacilli such as Mycoplasma and Legionella [2-3]. It also has antibacterial activity against Pseudomonas aeruginosa and Serratia [4]. These microorganisms are prone to resistance to beta-lactams. In recent years, along with the increased usage of these antibiotics, fluoroquinolone-resistant bacteria have also appeared.

Corresponding author: Dr. Mitsutoshi Satoh, Department of Toxicology and Pharmacology, Faculty of Pharmaceutical Sciences, Toho University. Miyama 2-2-1, Funabashi, Japan satoh@phar.toho-u.ac.jp 
In addition, multidrug - resistant Pseudo monasaeruginosa (MDRP) has become a significant problem. In 2003, MDRP-infectious disease was graded as category 5 by the infectious disease laws in Japan. Such disease is defined as $P$. aeruginosa showing resistance to beta-lactams, aminoglycosides and fluoroquinolones. Various attempts to control MDRP have been reported from overseas, including phage therapy, administration of colistin, and other antipseudomonal therapy [5]. However, these infections are more difficult to treat and rely on susceptibility reports to guide therapy, and iatrogenic infection and an increased rate of associated mortality have been reported in many hospitals in Japan.

It is one of the possibilities that inappropriate fluoroquinolone usage, especially doses that are too low, has resulted in an increase of MDRP infections. As proper use of CPFX is one of the most promising strategies for control of MDRP, it is important to ensure an optimal clinical outcome and prevent the emergence and spread of antimicrobial-resistant pathogens [6]. Administration methods based on pharmacokinetic/pharmacodynamic theory have been studied in relation to the proper usage of fluoroquinolones including CPFX. As CPFX is a concentration-dependent antibacterial drug, the AUC and Cmax are used as indices of efficacy. Recently, the AUC/minimum inhibitory concentration (MIC) ratio and Cmax/MIC ratio have also been used as indices of clinical efficacy in relation to the resistant properties of the target bacillus. Moreover, the AUC/MIC ratio is correlated closely with the antimicrobial activity of CPFX [7,8]. Optimal clinical and bacteriological responses in patients with lower respiratory tract infections are associated with the ability to achieve an AUC/MIC ratio of at least 125 [1]. However, many simulation studies have shown that the dosage and administration indicated in the CPFX package leaflet for the treatment of $P$. aeruginosa infections does not reach the target AUC/MIC ratio [9], hence, the potency of the antibacterial activity of CPFX may be insufficient at the commonly used dosage. The aim of this study was to investigate the use of CPFX in a hospital setting in order to obtain information that might help infection control teams to use antibiotics more appropriately, and prevent the emergence of drug resistance.

\section{MATERIALS AND METHODS}

\section{Study design}

This retrospective study was performed at Toho University Omori Hospital in Tokyo. After obtaining Institutional Review Board approval, we selected 112 adult patients diagnosed as having respiratory infections who had been treated as inpatients with i.v. CPFX for more than 3 days between November 2001 and March 2007. Exclusion criteria were known hypersensitivity to any of the drugs used in the study; pregnancy; lactating mothers; patients with severe impairment of renal function (serum creatinine $>2$ mg/dL); Methicillin-Resistant Staphylococcus Aureus (MRSA) infection; other antibiotic or antifungal therapy within one week.

\section{Definitions}

We used "The Japanese Respiratory Society guidelines" modified criteria to define MDRP infection severity and the efficacy of antibiotics (The Japanese Respiratory Society Guidelines) (Table 1) [10]. An MDRP isolate was defined as an organism that was resistant to at least three of the five antipseudomonal classes of antimicrobial agents. None of the patients received a monobactam. The Pseudomonas isolates were identified by the classic microbiologic method. Briefly, colonies compatible with $P$. aeruginosa (gram negative bacilli, glucose non-fermenting, oxidase-positive (usually with pyocyanin and distinctive odor) colonies) were submitted for to testing with the VITEK 2 system (fbioMérieux, France) for final identification. 
Table 1. Determination of the degree of severity of infection [10].

\begin{tabular}{|c|c|c|c|c|}
\hline & & M ild & M oderate & Sever \\
\hline Item s for judgem ent & & 4/7 applicab le & & 4/7 app licable \\
\hline Body tem perature & ('C) & $<37.5$ & $\begin{array}{l}\text { Corresponding to } \\
\text { ne ither } m \text { id nor }\end{array}$ & $\geqq 38.6$ \\
\hline Pulses & $(\mathrm{m}$ in $)$ & $<100$ & sever & $\geqq 130$ \\
\hline Resp iratory rates & $(\mathrm{m}$ in $)$ & $<20$ & & $\geqq 30$ \\
\hline Dehydration & & $(-)$ & $(-)$ or $(+)$ & $(+)$ \\
\hline Leukocyte & $\left(/ \mathrm{mm}^{3}\right)$ & $<10,000$ & - Corresponding to & $\geqq \underset{<4,000}{20,000 \text { or }}$ \\
\hline CRP & $(m \mathrm{~g} / \mathrm{dL})$ & $<10$ & $\begin{array}{c}\text { ne ither } \mathrm{m} \text { id nor } \\
\text { sever }\end{array}$ & $\geqq 20$ \\
\hline $\mathrm{SpO}_{2}$ & (\%) & & & $\leqq 90$ \\
\hline
\end{tabular}

CRP, C-reactive protein; $\mathrm{SpO}_{2}$, percutaneous oxygen saturation.

Minimum inhibitory concentration (MIC) was obtained from the antimicrobial susceptibility testing performed according to the guidelines of the Clinical and Laboratory Standards Institute (CLSI) [11]. Pneumonia was diagnosed as the presence of a new or progressive infiltrate, consolidation, or cavitation on chest radiographs. In addition, patients had to have a temperature of $>38^{\circ} \mathrm{C}$, leukopenia (leukocyte count, $<4,000 / \mathrm{mm}^{3}$ ), or leukocytosis (leukocyte count, $>12,000 / \mathrm{mm}^{3}$ ). The outcome of infection was assessed on day 6 as well as at the end of therapy, because this has been shown in a previous study to be the mean time until resolution of clinical parameters [5]. Patients who satisfied three or more of the following four items were considered to have received effective antibiotic treatment: (i) a temperature of $<37^{\circ} \mathrm{C}$ at the end of therapy, (ii) leukocyte count $<9,000 / \mathrm{mm}^{3}$ at the end of therapy, (iii) C-reactive protein (CRP) level at the end of therapy $<30 \%$ of that before CPFX treatment, and (iv) CT and X-ray evidence of improvement of the signs and symptoms of infection. The patients who satisfied less than three of these criteria were considered to have failed treatment.

\section{Calculation of pharmacokinetic parameters}

The creatinine clearance (Ccr) was calculated from the Cockcroft-Gault formula [12]: $\mathrm{Ccr}=$ $\{[140$ - age (years)] X weight $(\mathrm{kg})\}$ (X0.85 if female) $/\{72 \quad \mathrm{X} \quad$ [serumCr(mg/dL) $]\} . \quad$ The predicted plasma clearance (CL) [13] was calculated using the following formula: CL $(\mathrm{mL} / \mathrm{min})=$ weight $\mathrm{X}(0.167+0.00145 \mathrm{X} \mathrm{Ccr})$. The predicted AUC for individual patients was obtained from a modified formula reported by Forrest et al. (1993) [1]: AUC = dose (mg/day)/weight (kg) X $(0.167+0.00145 \mathrm{CL})$. Ideal body weight was calculated from the following formula: Ideal body weight $=50$ (45 if female) + 0.096 X (hight (cm) -152.4). Patient with equal or greater bodyweight/ideal bodyweight $(\mathrm{BMI} ; 22)=1.2$ were classified "obese", and AUC of the patients were calculated from ideal body weight and CL. For non-obese patients their total body weight was used.

\section{STATISTICAL ANALYSIS}

Data represent the mean \pm SD. Statistical analysis 
was assessed with the Student-Welch's tests for simple comparisons. All data were analyzed using Stat View ver 5.0 (SAS Institute, Japan).

\section{RESULTS}

\section{Study population and clinical data}

There were 112 patients (70 males and 42 females, height $159.0 \pm 9.7 \mathrm{~cm}$, body weight $51.7 \pm 13.8$ $\mathrm{kg}$, average age 67.9 years) for whom both pharmacokinetic and pharmacodynamic data were available, including 10 dialysis patients (8.9\%). Among the patients, 107 had pneumonia, 3 had bronchitis, one had a lung abscess and one had long-term infection. Sixteen of these patients had community-acquired pneumonia, and 96 had nosocomial pneumonia, including 16 patients with aspiration pneumonia. More than $80 \%$ of the patients treated with CPFX were classified as having moderate to severe MDRP.

\section{CPFX treatment and efficacy}

Ninety-nine patients received CPFX $300 \mathrm{mg}$ i.v. $\mathrm{q} 12 \mathrm{~h}$ as recommended by the package insert of CPFX injection provided in Japan. CPFX dosage a day was not adjusted by renal function of the patients (Figure 1). The efficacy rate was calculated for 111 patients excluding one patient with nosocomial pneumonia in whom no inflammatory reaction was revealed from the in laboratory data, although the patient was diagnosed as having pneumonia. The treatment was effective in 50 patients, and the percentage effectiveness was $44.6 \%$ (50/111 patients). The effectiveness for community-acquired pneumonia and nosocomial pneumonia was $75.0 \%$ (12/16 patients) and 40.0\% (57/95 patients), respectively.

\section{Isolated bacteria and frequency of isolation}

During CPFX treatment, cultures from the sites of infection were taken from 96 patients. MICs for CPFX were recorded in the medical chart of twenty-two patients attributed to $P$. aeruginosa. The study population included 11 patients (50.0\%) for whom the MICs were $0.5 \mathrm{mg} / \mathrm{L}$ or less, and 6 patients (26.1\%) for whom the MICs were more than $4 \mathrm{mg} / \mathrm{L}$ (drug-resistant strain).

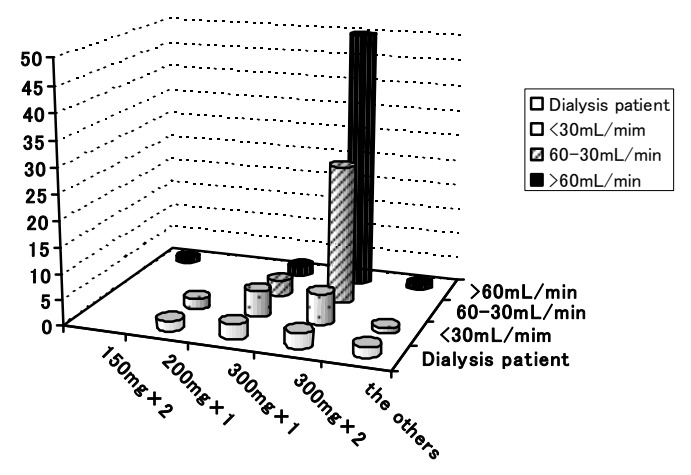

Figure 1. Distribution of CPFX dosage and differences of renal function.

\section{Pharmacokinetic parameters and efficacy of CPFX}

The association between probability of cure and pharmacokinetic/pharmacodynamic parameters (AUC and AUC/MIC value) for patients who had $P$. aeruginosa infections was analyzed. Other considered factors were body weight, height and laboratory data entered in the medical records. The association between probability of cure and AUC/MIC value for 19 patients was analyzed. The AUC/MIC ratio calculated from the predicted AUC for individual patients was $37.2 \pm 41.6$ and $87.8 \pm 23.1$ for failures and clinical cures, respectively. The difference between the two ratios was significant $(\mathrm{p}=0.0035)$ (Table 2). Both AUC/MIC ratio for were considerably lower than the target value of 125 for gram-negative aerobes.

The association between probability of cure and AUC value for 94 patients was then analyzed. The predicted AUC for individual patients mentioned under Methods was $42.2 \pm 19.0$ $\mathrm{mg} \cdot \mathrm{min} / \mathrm{mL}$ and $48.6 \pm 19.4 \mathrm{mg} \cdot \mathrm{min} / \mathrm{mL}$ for failures and clinical cures, respectively (Table 2). 
Table 2. Efficacy and pharmacokinetic/pharmacodynamic parameters of CPFX for patients who had $P$. aeruginosa infections.

\begin{tabular}{|c|c|c|c|c|c|c|}
\hline & & M ean $\pm S . D$. & $\mathbf{N}$ & Range & $95 \%$ C I & $P$-value \\
\hline \multirow{2}{*}{ AUC / M IC } & Cures & $87.8 \pm 23.1$ & 7 & $60.9-123.0$ & $66.4-109.2$ & \multirow{2}{*}{0.0035} \\
\hline & Failures & $37.2 \pm 23.1$ & 12 & $1.1-154.2$ & $10.8-63.7$ & \\
\hline \multirow{2}{*}{$\begin{array}{l}\text { AUC } \\
(m g \mathrm{~m} \mathrm{in} / \mathrm{mL})\end{array}$} & Cures & $48.6 \pm 19.4$ & 42 & $22.5-113.3$ & $42.5-54.6$ & \multirow{2}{*}{0.0342} \\
\hline & Failures & $42.2 \pm 19.0$ & 52 & $15.8-87.7$ & $36.9-47.5$ & \\
\hline \multirow{2}{*}{$\begin{array}{l}\text { Ccr } \\
(m L / m \text { in) }\end{array}$} & Cures & $69.3 \pm 36.8$ & 40 & $12.2-147.6$ & $59.6-79.3$ & \multirow{2}{*}{0.0686} \\
\hline & Failures & $82.0 \pm 43.1$ & 41 & $11.0-170.3$ & $68.4-95.6$ & \\
\hline \multirow{2}{*}{$\begin{array}{l}\text { Body we ight } \\
(\mathrm{kg})\end{array}$} & Cures & $51.5 \pm 12.0$ & 40 & $24.1-75.0$ & $47.7-55.3$ & \multirow{2}{*}{0.3379} \\
\hline & Failures & $52.9 \pm 16.6$ & 41 & $29.1-98.0$ & $47.6-58.1$ & \\
\hline
\end{tabular}

The associations between probability of cure and body weight and predicted Ccr value for the 81 patients who received CPFX 300 mg i.v. q12h were also analyzed. The predicted Ccr for individual patients mentioned in the Methods was $82.0 \pm 43.1 \mathrm{~mL} / \mathrm{min}$ and $69.3 \pm 36.8 \mathrm{~mL} / \mathrm{min}$ for failures and clinical cures, respectively (Table 2). Urinary function in the clinically cured patients tended to be poorer than in the patients for whom treatment failed $(\mathrm{p}=0.0686)$.

\section{DISCUSSION}

In the present study, the clinical use of CPFX for the treatment of infectious diseases does not reach the target AUC/MIC ratio, and that the concentration of CPFX is not within the range to which many pathogens are susceptible in a large proportion of patients. It is important to monitor the blood concentration of CPFX based on pharmacodynamic parameters (Cmax, AUC, AUC/MIC ratio) to ensure the effective treatment of patients with infectious diseases and to prevent the development of resistance in bacteria.

Although CPFX is a drug that is excreted mainly into urine, 96 patients including patients with renal dysfunction received CPFX $300 \mathrm{mg}$ i.v. q12h without any consideration of individual patient age, weight, renal function, or other characteristics. When clinicians prescribe CPFX, no standard regimen exists. For this reason, it is possible that a low blood concentration of CPFX that cannot be expected to have antibiotic effectiveness will occur in patients with normal renal function. Therefore, it is reasonable to speculate that drug resistance will arise if inappropriate doses are administered in this situation. In this study, we confirmed that the AUC/MIC ratio calculated from the predicted AUC for individual patients who achieved clinical cure was significantly higher than that in patients for whom treatment failed. Although it is reported that the target value of the AUC/MIC ratio for $P$. aeruginosa is commonly more than $125[14,15]$, the average AUC/MIC ratio for clinical cure was about 90 in our study, and the 
target achievement ratio for $P$. aeruginosa was $4.5 \%(1 / 22)$. These results suggest that the target value of the AUC/MIC ratio for $P$. aeruginosa should be reconsidered in the clinical use. The relationship between the efficacy of CPFX and the AUC/MIC ratio was closer than the relationship between efficacy and AUC, suggesting that it is important to adjust the dose in consideration of the MIC for infectious bacteria. Furthermore, the relationship between efficacy and Ccr needs to be taken into consideration because the AUC of CPFX is influenced by renal function. Although CPFX is an antibiotic that is secreted into urine, adjustment of the CPFX dose according to differences in renal function is not performed, and CPFX is almost always administered by the standardized regimen to all patients. The blood concentration of CPFX in patients with normal renal function is relatively lower than in patients with renal dysfunction, and this might reduce the efficacy in patients with normal renal function. Montgomery et al. (2001) [9] reported that $1200 \mathrm{mg} /$ day is an acceptable CPFX dose. And CPFX 400mg i.v. q8h is approved by FDA for Pseudomonas infections. Therefore the dose of $600 \mathrm{mg} /$ day that is usually used should be increased or an additional agent employed. Furthermore, it is necessary to clarify whether a low dose of CPFX leads to tolerance to antibiotics from the viewpoint of the mutant selection window (MSW), and it is necessary to adjust the most suitable dosage for each individual patient. And it is important to lead to higher Cmax/MIC ratio by the regimen of CPFX i.v. once daily, which is associated with a more significant postantibiotic effect and higher efficacy of fluoroquinolones [16]. To develop the best administration plan for each patient, implementation of the TDM is indispensable.

CPFX is expected to be effective in patients who do not respond to other antimicrobials or have severe infection. Our studies revealed that the standard regimen may not take the characteristics of CPFX into consideration in many cases. Since dose adjustment of CPFX according to differences in renal function was not performed in the present study, differences in efficacy of the same dose due to renal function may occur. In several clinical studies from other countries, insufficient dosage has been reported [9,14,15]. Although a high dosage of CPFX in Japan has not been reported, clinical doses that are double the Japanese dose are usually used in other countries. The average value of the AUC/MIC ratio (90) for clinical cure in our study was different from the target value of 125 for $P$. aeruginosa. Taking the renal function of patients and the MIC of the causative organisms into consideration, appropriate usage of antibiotics by implementation of the TDM will help to prevent the spread of tolerance to antibiotics and maintain their effectiveness.

\section{ACKNOWLEDGMENT}

The authors thank Dr. Edward E. Knaus for careful and continuous editorial support. The authors greatly appreciate the significant scientific contribution of Dr. Ronald G. Micetich.

\section{REFERENCES}

[1]. Forrest, C.H. Ballow, D.E. Nix, M.C. Birmingham, J.J. Schentag, Development of a population pharmacokinetic model and optimal sampling strategies for intravenous ciprofloxacin. Antimicrob. Agent Chemother., 37, 1065-1072 (1993).

[2]. J. Sander, Fluoroquinolones--a new and important group of antimicrobial agents. Tidsskr Nor Laegeforen, 112, 346-349 (1992).

[3]. R.J. Fallon, W.M. Brown, In-vitro sensitivity of legionellas, meningococci and mycoplasmas to ciprofloxacin and enoxacin. $J$ Antimicrob Chemother., 15, 787-789 (1985).

[4]. W. Klietmann, J. Focht, K. Nösner, Comparative in vitro activity of ciprofloxacin against aerobic and anaerobic bacteria from clinical isolates. Arzneimittelforschung, 37, 661-666 (1987). 
[5]. R. Reina, E. Estenssoro, G. Sa'enz, H.S. Canales, R. Gonzalvo, G. Vidal, G. Martins, A. Das Neves, O. Santander, Safety and efficacy of colistin in Acinetobacter and Pseudomonas infections: a prospective cohort study. Intensive Care Med., 31, 1058-1065 (2005).

[6]. G. Höffken, M.S. Niederman, Nosocomial pneumonia: the importance of a de-escalating strategy for antibiotic treatment of pneumonia in the ICU. Chest., 122, 2183-2196 (2002).

[7]. M.M. Sánchez-Recio, C.I. Colino, A. Sánchez-Navarro, A retrospective analysis of pharmacokinetic/pharmacodynamic indices as indicators of the clinical efficacy of ciprofloxacin. J Antimicrob Chemother., 45, 321-328 (2000).

[8]. C.E. Thorburn, D.I. Edwards, The effect of pharmacokinetics on the bactericidal activity of ciprofloxacin and sparfloxacin against Streptococcus pneumoniae and the emergence of resistance. J Antimicrob Chemother., 48, 15-22 (2001).

[9]. M.J. Montgomery, P.M. Beringer, A. Aminimanizani, S.G. Louie, B.J. Shapiro, R. Jelliffe, M.A. Gill, Population Pharmacokinetics and use of Monte Carlo Simulation to evaluate Currently recommended dosing Regiments of ciprofloxacin in adult patients with cystic fibrosis. Antimicrob. Agent Chemother., 45, 3468-3473 (2001).

[10]. S. Kohno, T. Matsushima, A. Saito, K. Nakata, K. Yamaguchi, A. Watanabe, N. Aoki, H. Goto, K. Suzuki, K. Sekizawa, K. Soma, T. Nagatake, K. Mikasa, The Japanese Respiratory Society guidelines for management of respiratory infections (2002). The Japanese Respiratory Society.

[11]. Clinical and Laboratory Standards Institute. Methods for antimicrobial susceptibility testing of anaerobic bacteria; approved standard, seventh edition. M11-A7. Wayne, PA: CLSI (2007).

[12]. D.W. Cockcroft, M.H Gault., Prediction of Creatinine from Serum Creatinine. Nephron, 16, 31-41 (1976).
[13]. A. Forrest, D.E. Nix, C.H. Ballow, T.F. Goss, M.C. Birmingham, J.J. Schentag, Pharmacodynamics of intravenous Ciprofloxacin in Seriously ill patients. Antimicrob. Agent Chemother., 37, 1073-1081 (1993).

[14]. A.A. Firsov, S.H. Zinner, I.Yu Lubenko, S.N. Vostrov, Gemifloxacin and ciprofloxacin pharmacodynamics in an in-vitro dynamic model: prediction of the equivalent AUC/MIC breakpoints and doses. Int $J$ Antimicrob Agents., 16, 407-414 (2000).

[15]. M.W. Garrison, Pharmacodynamic assessment of the activity of high-dose (750 mg) levofloxacin, ciprofloxacin, and gatifloxacin against clinical strains of Pseudomonas aeruginosa. Diagn Microbiol Infect Dis., 54, 51-56 (2006).

[16]. M.D. Sánchez Navarro, C. Coloma Milano, A. Zarzuelo Castañeda, M.L. Sayalero Marinero, A. Sánchez-Navarro, Pharmacokinetics of ciprofloxacin as a tool to optimise dosage schedules in community patients. Clin Pharmacokinet., 41, 1213-1220 (2002). 